'New' and 'old' antidepressants: all equal in the eyes

\section{of the lore?*}

GORDON PARKER

Ever since their introduction, it has been widely promulgated that all antidepressants are equally effective. In light of their differing pharmacological profiles, an 'all roads lead to Rome' model challenges our mechanistic understanding of 'depression'. While many clinicians mutter hesitantly that some of the newer antidepressants appear less effective, such impressionistic views are at variance with psychiatry's largest evidence base. If it does not capture 'the truth', then the phrase 'evidence-based psychiatry' risks oxymoron status. Thus, this editorial explores difficulties in reconciling efficacy data with clinical observation, focusing on two major classes of older and newer antidepressants - tricyclics (TCAs) and selective serotonin reuptake inhibitors (SSRIs).

\section{COMPARATIVE EFFICACY DATA}

Anderson (2000) analysed efficacy data from 102 randomised controlled studies of TCAs and SSRIs involving more than 10000 patients, determining no overall efficacy difference (effect size $=-0.03$, $95 \%$ CI -0.09 to 0.03$)$. Williams et al (2000) considered 150 studies involving more than 160000 participants with major depression. The grouped data indicated that $54 \%$ of both those receiving an old and a new antidepressant improved by at least $50 \%$ (relative benefit $1.0,95 \%$ CI $0.97-$ 1.06). They also considered 38 studies (involving 4064 patients) directly comparing SSRIs and TCAs, again finding no significant outcome difference. Such seemingly

\footnotetext{
* This editorial is an expansion and update of one of five issues addressed in the 1999 William Sargant Memorial Lecture,'Dysfunctional assumptions about the depressive disorders', delivered to the Royal College of Psychiatrists' Faculty for General and Community Psychiatry and the CollegiateTrainees' Committee, 21 January 1999, Nottingham, UK.
}

unequivocal results argue for the new and old antidepressants being equally efficacious and the TCAs and SSRIs equipotent.

\section{DO INCONSISTENCIES IN THE EFFICACY DATABASE INFORM?} there was no efficacy difference between the TCAs and SSRIs in general practice significantly superior within the in-patient group-where Anderson speculated influence of a more severe depression, a greater chance of melancholia or suicide risk, or factors independent of patient status - such as the pharmacology of TCAs. As in-patient status and more severe depression may be proxies for melancholia, the in-patient subsample differential is potentially informative.

\section{ARE SSRIS LESS EFFICACIOUS IN MELANCHOLIA?}

The Danish University Antidepressant Group (DUAG) was the first to challenge the comparable efficacy Zeitgeist, conducting separate double-blind studies of clomipramine and two SSRIs, citalopram (DUAG, 1986) and paroxetine (DUAG, 1990), with the TCA being more effective than the SSRIs in the overall samples. Results are commonly misinterpreted (e.g. Perry, 1996) as indicating that the TCA was superior for melancholic depression, but more argue the converse. Thus, while the DUAG data indicate that endogenous (qua melancholic) patients were twice as likely to respond to a TCA than to an SSRI, non-endogenous patients showed an even greater likelihood (i.e. 5-7 times) of responding to the TCA.

Ignoring open studies, Amsterdam (1998) identified, in addition to the DUAG ones, four comparison studies supposedly
In the Anderson (2000) meta-analysis, and out-patients, but the TCAs were demonstrating that the SSRIs are comparably less efficacious for melancholic depression. In fact, all bar one determined comparable response rates, with only the non-randomised study by Roose et al (1994) of 54 elderly patients revealing a clear trend (with $83 \%$ and $10 \%$ response rates to nortriptyline and fluoxetine, respectively).

\section{EFFECTIVENESS STUDIES}

Effectiveness studies essentially evaluate treatments in conditions corresponding to standard clinical practice. Colleagues and I have undertaken both retrospective and longitudinal effectiveness studies. The first (Parker et al, 1999) comprised 369 patients with depression evaluating previous antidepressant treatments. Mean sample effectiveness ratings were slightly higher for TCAs than for SSRIs (i.e. $1.10 v .0 .89$ ). For those meeting DSM-IV (American Psychiatric Association, 1994) melancholic criteria, ratings distinctly favoured TCAs over SSRIs (i.e. $1.10 v$. 0.66), but were comparable (i.e. $1.03 v$. 1.01) for patients with non-melancholic depression. In a second, 'naturalistic' study (Parker et al, 2001), 182 patients with depression were reassessed after 12 months, with $34 \%$ rating their TCA as 'effective' compared with $29 \%$ of those receiving an SSRI. For those meeting DSM-IV melancholia criteria, $48 \%$ receiving a TCA met effectiveness criteria (compared with $20 \%$ of those receiving an SSRI), while for residual non-melancholia patients, respective effectiveness ratings were $25 \%$ and $33 \%$.

Study consistency was striking - in indicating that TCAs and SSRIs appeared similarly effective in non-melancholic depression, and that TCAs appeared superior for melancholic depression, with overall group differences 'driven' by the representation of subjects with melancholic depression.

\section{WHY THE DISCORDANCE BETWEEN EFFICACY STUDIES AND CLINICAL OBSERVATION?}

Efficacy studies inform us whether a particular drug is efficacious - information necessary to licensing authorities and to clinicians, but not sufficient. Most industrysponsored efficacy studies of antidepressant 
drugs involve out-patients whose depression is, at worst, of modest severity, which risks losing representation of those with the melancholic depressive subtype; furthermore, examining only grouped data may hide subgroup efficacy nuances.

If a new antidepressant is comparatively ineffective for (say) melancholia, it would take years before its 'pattern' crystallised even for observant clinicians. Identification would be further held back by modelling depression as a dimensional unitary concept, by difficulties in measuring 'melancholia' validly and by having treatment guidelines and opinion leaders opine that "all antidepressants are equally effective".

Such circumstances prevailed for the first decade following the introduction of SSRIs. Doubts are now emerging, as evidenced in the recent American Psychiatric Association (2000) Practice Guidelines, where it is noted that

"Some studies have also suggested that in major depressive disorder marked by melancholic features, tricyclic antidepressants may be additionally effective . . . as well as superior to SSRIs".

This is the first official 'crack' in the edifice that all antidepressants are equally efficacious (qua effective).

\section{IMPLICATIONS OF DIFFERENTIAL EFFECTIVENESS}

As broad-based antidepressants TCAs may be more effective than SSRIs. However, while SSRIs appear of comparable effectiveness to TCAs for non-melancholic depression, SSRIs may be less effective in melancholic depression. If this is the case, there are several implications and consequences.

First, we need to review current clinical management strategies. Accepting the evidence, psychiatrists increasingly prescribe a new antidepressant and, if it fails, trial several other new antidepressants, whether of the same class or not. Accepting views that older drugs have more sideeffects and are dangerous, many Western psychiatrists are now reluctant to prescribe them, and electroconvulsive therapy is often contemplated as the next logical strategy when newer antidepressants fail. We need to revisit the utility of the older antidepressants.

This does not mean, of necessity, that the SSRIs should not be prescribed for

GORDON PARKER, FRANZCP, School of Psychiatry, University of New South Wales, Kiloh Centre, Prince of Wales Hospital, Randwick 203I, Australia

(First received I8 August 2000, final revision I2 February 200I, accepted I3 February 200I)

melancholic depression, particularly if there has been a previous response, the episode is an initial one and, anecdotally, if the patient is young and if observable psychomotor disturbance is absent or slight. It might suggest, however, that any such trial might be relatively brief and, if no improvement is observed, consideration should be given to a more broad-based antidepressant.

Second, we require more efficacy data from clinical panel studies, and for treatment guidelines to both move away from their focus on efficacy data and respect the importance of depressive subtypes, rather than operate to a dated and limited severity-based paradigm (Parker, 2000).

Third, should the pharmaceutical companies be apprehensive? Yes, if their antidepressant is truly less effective as an antidepressant or if they view their drug as being all things to all depressions - rather than identifying its niche by examining the strengths and weaknesses of the drug across differing conditions. If, as suggested here for SSRIs, any (overall) decreased effectiveness is restricted to melancholic depression, this should not be a substantive concern - as melancholia is (comparatively) of low prevalence. If equipotent for non-melancholic depression, then obvious cost-benefit advantages of SSRIs (e.g. adherence, side-effect profile) remain. More importantly, the industry might then seek to determine what properties of the SSRI are missing (in comparison to the older broad-based antidepressants) that are relevant to the pathogenesis of melancholia, and thus 'boutique' drugs might emerge more rapidly. There is much to be gained by the adoption of a new model that rejects views that all depressive disorders are the same and that the antidepressants have comparable levels of effectiveness.

\section{ACKNOWLEDGEMENTS}

Thanks to colleagues at the Mood Disorders Unit for their comments, and Kerrie Eyers for manuscript assistance.

\section{DECLARATION OF INTEREST}

None relevant. I have received no honoraria or sponsorship from any company involved in the manufacture of the older antidepressants.

\section{REFERENCES}

American Psychiatric Association (1994) Diagnostic and Statistical Manual of Mental Disorders (4th edn) (DSM-IV). Washington, DC: APA.

- (2000) Practice Guidelines for the Treatment of Psychiatric Disorders. Compendium 2000. Washington, DC: American Psychiatric Association.

Amsterdam, J. D. (1998) Selective serotonin reuptake inhibitor efficacy in severe and melancholic depression. Journal of Psychopharmacology, 12 (suppl. B), S99-SIII.

Anderson, I. M. (2000) Selective serotonin reuptake inhibitors versus tricyclic antidepressants: a metaanalysis of efficacy and tolerability. Journal of Affective Disorders, 58, 19-36.

Danish University Antidepressant Group (1986) Citalopram: clinical effect profile in comparison with clomipramine. A controlled multicenter study. Journal of Psychopharmacology, 90, I31-138.

_ (1990) Paroxetine: a selective serotonin reuptake inhibitor showing better tolerance, but weaker antidepressant effect than clomipramine in a controlled multicenter study. Journal of Affective Disorders, $\mathbf{1 8}$, 289-299.

Parker, G. (2000) Classifying depression: should paradigms lost be regained? American journal of Psychiatry, 157, |195-1203.

_, Mitchell, P., Wilhelm, K., et al (1999) Are the newer antidepressant drugs as effective as established physical treatments? Results from an Australasian clinical panel study. Australian and New Zealand journal of Psychiatry, 33, 874-88I.

_, Roy, K., Wilhelm, K., et al (200I) Assessing the effectiveness of differing antidepressant therapies: a prospective clinical practice study. Journal of Clinical Psychiatry, 62, 117-125.

Perry, P. J. (1996) Pharmacotherapy for major depression with melancholic features: relative efficacy of tricyclic versus selective serotonin reuptake inhibitor antidepressant. Journal of Affective Disorders, 39, I-6.

Roose, S. P., Glassman, A. H., Attia, E., et al (1994) Comparative efficacy of selective serotonin reuptake inhibitors and tricyclics in the treatment of melancholia. American Journal of Psychiatry, I5I, 1735-1739.

Williams, J. W., Jr, Mulrow, C. D., Chiquette, E., et al (2000) A systematic review of newer

pharmacotherapies for depression in adults: evidence report summary: Clinical Guidelines, Part 2. Annals of Internal Medicine, I32, 743-756. 